\title{
Cell-Free Protein Synthesis From Fast-Growing Vibrio natriegens
}

\author{
Jurek Failmezgert, Steffen Scholz ${ }^{\dagger}$, Bastian Blombach and Martin Siemann-Herzberg* \\ Institute of Biochemical Engineering, University of Stuttgart, Stuttgart, Germany
}

Vibrio natriegens constitutes one of the fastest-growing nonpathogenic bacteria and a potential novel workhorse for many biotechnological applications. Here, we report the development of a Vibrio-based cell-free protein synthesis system (CFPS). Specifically, up to $0.4 \mathrm{~g} \mathrm{~L}^{-1}$ eGFP could be successfully synthesized in small-scale batch reactions using cell-free extract obtained from fast-growing $V$. natriegens cultures. Versatile CFPS system characterization attained by combining the analyses of key metabolites for translation and ribosomes revealed limitations regarding rRNA stability and critical

OPEN ACCESS

Edited by:

Jörg Stülke,

Georg-August-Universität Göttingen,

Germany

Reviewed by:

Gert Bange,

Philipps University of Marburg,

Germany

Pablo Ivan Nikel,

The Novo Nordisk Foundation Center for Biosustainability (DTU Biosustain),

Denmark

*Correspondence:

Martin Siemann-Herzberg siemann@ibvt.uni-stuttgart.de

tThese authors have contributed equally to this work.

Specialty section:

This article was submitted to Microbial Physiology and Metabolism, a section of the journal Frontiers in Microbiology

Received: 24 April 2018 Accepted: 14 May 2018

Published: 01 June 2018

Citation:

Failmezger J, Scholz S, Blombach B and Siemann-Herzberg M (2018) Cell-Free Protein Synthesis From Fast-Growing Vibrio natriegens. Front. Microbiol. 9:1146. doi: 10.3389/fmicb.2018.01146 substrate consumption (e.g., amino acids). Alternatively, rRNA showed increased stability by inducing $\mathrm{Mg}^{2+}$ homeostasis in the reaction. Although the enormous translation capacity of the CFPS system based on the available ribosome concentration could not yet be fully exploited, its potential was successfully demonstrated by activating an endogenous transcription unit with $V$. natriegens RNA polymerase (RNAP) for protein expression. This allowed the use of in vitro screening for promoter strength, a critical factor for efficient gene expression in vitro and in vivo. Three different promoters were tested and output signals corresponded well with the expected affinity for $V$. natriegens RNAP. This established CFPS toolbox may provide a foundation to establish $V$. natriegens as a valuable platform in biotechnology as well as synthetic biology.

Keywords: in vitro translation, Vibrio natriegens, ribosomes, rRNA, cell-free extract

\section{INTRODUCTION}

Recent studies highlight the potential of fast-growing bacteria to speed up lab routines and biotechnological processes (Cordova et al., 2015; Weinstock et al., 2016; Hoffart et al., 2017). Among such bacteria, the Gram-negative $\gamma$-proteobacterium Vibrio natriegens represents a promising candidate that possesses remarkably high growth and substrate consumption rates (Weinstock et al., 2016; Hoffart et al., 2017; Long et al., 2017) as prerequisite to develop fermentation processes operating with very high productivity (Hoffart et al., 2017) Furthermore, genetic engineering tools for $V$. natriegens have been developed and allow the directed manipulation of its cellular metabolism (Weinstock et al., 2016; Hoffart et al., 2017) and heterologous protein production (Weinstock et al., 2016). In particular, inducible promoter systems (e.g., lacUV5, trc, and $a r a B A D$ ) have been validated for the latter to drive expression of $g f p$ in V. natriegens (Weinstock et al., 2016).

$V$. natriegens grows rapidly in minimal medium supplemented with various carbon sources under both aerobic and anaerobic conditions (Hoffart et al., 2017). However, exceptionally fast growth was observed in complex medium (Eagon, 1962; Weinstock et al., 2016; Hoffart et al., 2017) 
reaching differential growth rates of up to $4.43 \mathrm{~h}^{-1}$, which corresponds to a doubling time $\left(t_{d}\right)$ of 9.4 min (Hoffart et al., 2017). Aiyar et al. (2002) showed that with increasing growth rate the increase in ribosome number in $V$. natriegens accelerates yielding an estimated number of 115,000 ribosomes per cell at a $t_{d}$ of approximately $15 \mathrm{~min}$. This number is significantly higher compared to 70,000 ribosomes per cell in Escherichia coli at a $t_{d}$ of $25 \mathrm{~min}$ and also outcompetes the extrapolated value of 90,000 ribosomes per cell at a $t_{d}$ of 15 min (Aiyar et al., 2002). These characteristics support that $V$. natriegens may have potential as a platform for cell free protein synthesis (CFPS).

Cell-free systems, originated from crude cell extracts predominantly derived from $E$. coli, have been successfully used to discover the mechanisms of life, such as deciphering the genetic code or protein synthesis (for a recent review see Moore et al., 2017b). This technology has also been established in many fields of applied biotechnology, thereby enabling new applications in genetic prototyping and biomanufacturing. CFPS has been applied as well in the upcoming disciplines of synthetic biology, such as for the rapid prototyping of biological circuits and metabolic pathways with the long-term goal of using CFPS for natural product discovery (Takahashi et al., 2015; Jiang et al., 2018). Moreover, the design of paperbased cell-free diagnostics (Pardee et al., 2014, 2016) broadens its potential application in pharmaceutical diagnostics. In particular, because of the absence of barriers such as the cell wall or membranes, the cell-free reaction environment is open and therefore accessible and controllable, thus allowing direct and easy manipulation, monitoring, sampling, and optimization (Jewett et al., 2008). Notably, these features idealistically encompass basic experimental requirements; e.g., for detailed reaction analysis in systems biology (Niess et al., 2017).

Recently some E. coli-alternative CFPS systems were successfully introduced. Streptomyces-based systems were reported to address antibiotic production-related issues (Li et al., 2017; Moore et al., 2017a), and certain synthetic biology aspects (promoter-studies) were addressed using Bacillus subtilis as a core system (Kelwick et al., 2016). Moreover, several systems based on crude extract from eukaryotic cells, namely from Saccharomyces cerevisiae (Hodgman and Jewett, 2013; Gan and Jewett, 2014), rabbit reticulocytes (mammalian) (Anastasina et al., 2014), and Chinese hamster ovary (CHO, mammalian) cells (Brödel et al., 2014) were established. These systems offer the advantage of various degrees of posttranslational modifications but are restricted to low productivity, most likely owing to the rather low level of translationally active compounds such as ribosomes. This suggests that highly productive protein synthesis may instead be compiled using ultra-fast-growing (non-pathological) microbes, targeting Vibrio as a most promising candidate considering its unparalleled growth rate and high ribosome number. In addition, a robust cell-free Vibrio-based system may also serve as a promising platform to complement existing strategies for pathway discovery. In this context, we present the development and characterization of a CFPS system using crude extract derived from $V$. natriegens.

\section{RESULTS AND DISCUSSION}

\section{Vibrio natriegens Supports CFPS}

Our goal was to set up a CFPS system using fast-growing $V$. natriegens biomass by adopting the well-established protocols available for $E$. coli in vitro translation. These protocols usually consist of biomass cultivation via a bioreactor or shake flask, harvest of the biomass during fast growth, cell lysis via highpressure homogenization, high-speed centrifugation, run-off reaction, dialysis, and a final centrifugation step (Pratt, 1984; Nevin and Pratt, 1991; Liu et al., 2005). Even though we have recently demonstrated active cell-free extract from nongrowing cells (Failmezger et al., 2017b), we decided to utilize the traditional cell-free extract preparation method as described above. An exception was the growth medium: instead of using $2 \times$ YTPG, which is the most applied media for cell-free translation systems using E. coli, V. natriegens was cultivated in BHN (brainheart infusion with $\mathrm{NaCl}$ ). In this manner, high specific growth rates up to $3 \mathrm{~h}^{-1}$ were obtained in shake flask cultivations performed at $37^{\circ} \mathrm{C}$ (Figure 1A).

The biomass was harvested at maximum growth after only $2 \mathrm{~h}$ of cultivation and cell-free extract was prepared as described earlier in the text. To test the potential of in vitro translation for the cell-free extract from $V$. natriegens, we commenced by performing cell-free protein synthesis of eGFP. We used the identical reaction setup as described in our previous report for E. coli (Failmezger et al., 2016). In detail, transcription of eGFP was controlled by a T7 promoter and the reaction was spiked with T7 RNA polymerase (RNAP). Concentrations of substrates (amino acids and nucleotides), salts, and buffer components were also adopted. Exogenous energy regeneration by creatine phosphate and creatine kinase was used to supply the system with regenerated ATP and GTP. The reaction was carried out in a scale of $250 \mu \mathrm{L}$ at $37^{\circ} \mathrm{C}$, and eGFP synthesis was monitored online. Notably, combined transcription and translation in the $V$. natriegens system was confirmed by eGFP synthesis as demonstrated in Figure 1B. Expression of eGFP was detected after 30 min of incubation and was accompanied by a linear increase of fluorescence over the course of about $1 \mathrm{~h}$. Active synthesis was followed by a decline of the synthesis rate and a termination of the reaction after a total reaction time of $2 \mathrm{~h}$.

\section{Characterization of the $V$. natriegens Cell-Free Extract}

Upon demonstration of active protein synthesis by a cell-free translation system based on $V$. natriegens, our aspiration was to examine and characterize the system. As ribosomes denote the key for translation, we aimed to quantify the rRNA concentration in the extract. Extraction and quantification of rRNA revealed a concentration of $113 \pm 6 \mathrm{~g} \mathrm{~L}^{-1}$ rRNA (sum of $16 \mathrm{~S}$ and 23S) in the cell-free extract from $V$. natriegens. This concentration is roughly four-times higher than that in our standard E. coli cellfree system and mirrors the observed fast growth of $V$. natriegens. Moreover, the measured rRNA concentration correlates nicely with the approximately four-fold higher specific growth rate between $V$. natriegens and $E$. coli at the point of cell harvest 

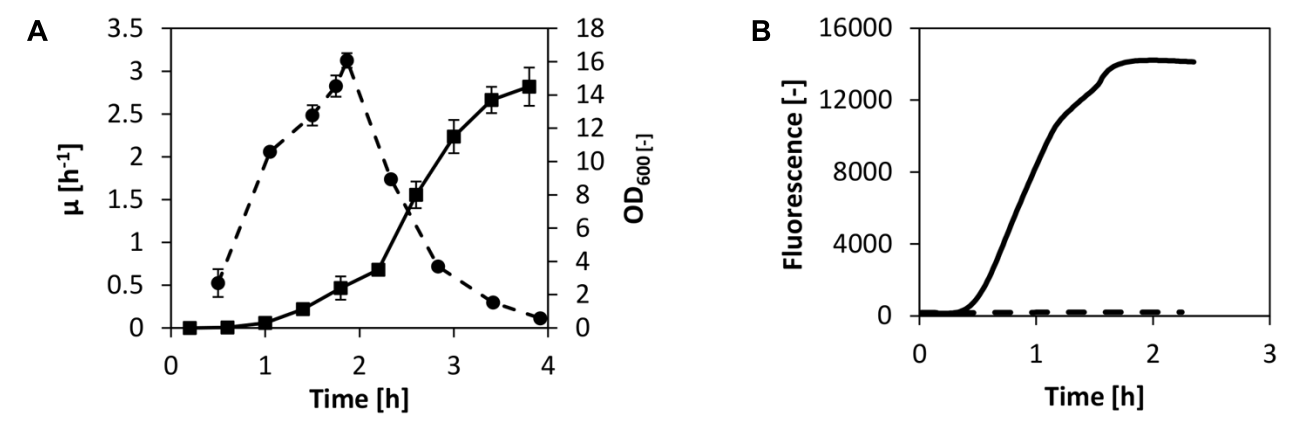

FIGURE 1 | CFPS from V. natriegens. (A) Shake flask cultivation of $V$. natriegens in $\mathrm{BHIN}$ media at $37^{\circ} \mathrm{C}$. Depicted are the biomass (solid line) and the differential specific growth rate (dashed line). For lysate preparation, cells were harvested at maximum growth. Error bars denote standard deviations of three independent measurements. (B) Cell-free protein synthesis of eGFP using V. natriegens cell-free extract (solid line). The negative control without plasmid is also shown (dashed line).

and is consistent with the general consensus that $V$. natriegens achieves fast growth through increased ribosome numbers (Aiyar et al., 2002). In addition, as we always resuspended $1 \mathrm{~g}$ biomass (wet weight) in $1 \mathrm{~mL}$ lysis buffer prior to homogenization, the direct comparison of certain characteristics, such as the ribosome concentration between both systems, is justified.

CFPS depends on highly active catalytic proteins provided by the cell-free extract. However, although we have previously shown that the cell-free extract preparation procedure preserves crucial components involved in translation in the case of E. coli (Failmezger et al., 2016), measurement of the rRNA integrity in the $V$. natriegens system before and after the run-off incubation at $37^{\circ} \mathrm{C}$, which can be regarded as the most critical step of the process, revealed no alteration in the rRNA integrity or concentration (Supplementary Figure S1). Hence, we concluded that similar to E. coli, lysate processing conditions do not detrimentally affect the catalyst composition.

\section{Efficient Energy Regeneration by Exogenous but Not Endogenous Systems}

Supplying the transcription and translation reactions with energy is crucial for efficient protein synthesis (Kim and Swartz, 1999; Kim and Kim, 2009). Therefore, we compared several of the most common energy regeneration systems, namely creatine phosphate in combination with creatine kinase, phosphoenolpyruvate (PEP), and pyruvate, for their ability to energize the translation reaction in the $V$. natriegens CFPS system. For each system, we optimized the magnesium ion concentration, a parameter well known to impact the translation performance (Kim and Choi, 2000; Failmezger et al., 2016). Similar to cell-free translation systems from other source organisms; e.g., from B. subtilis (Kelwick et al., 2016), we found that the magnesium concentration heavily influenced the synthesis rate in the CFPS from $V$. natriegens. This is demonstrated in Figure 2, which illustrates the relative synthesis rate at different concentrations of magnesium at a constant concentration of $60 \mathrm{mM}$ creatine phosphate. A clear optimum at $18 \mathrm{mM}$ magnesium was achieved. Notably, the

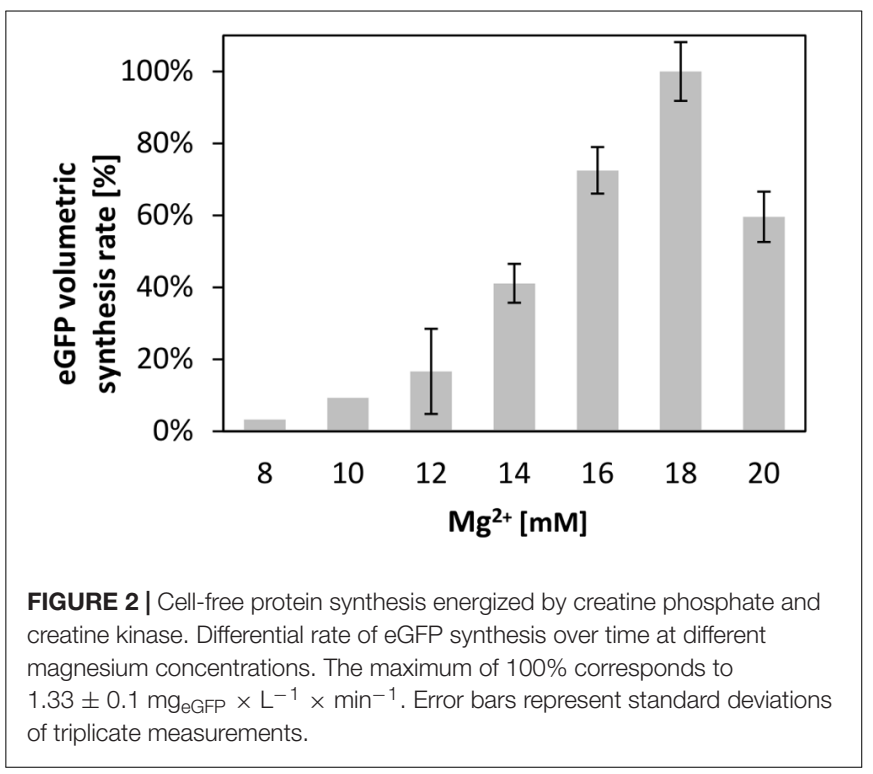

increase of the maximum rates, as well as the eGFP titer, both correlated linearly with the Mg-glutamate concentration until the optimum was reached $\left(R^{2}=0.95\right.$ or $R^{2}=0.93$ for a linear regression).

Next, we investigated the potential of endogenous energy regeneration by supplying the reaction with PEP or pyruvate. We observed active translation using PEP; however, the eGFP synthesis rates were markedly decreased compared to those of creatine phosphate (Supplementary Figure S2A). A similar scenario was obtained using pyruvate to regenerate ATP, with similar final titers and maximum synthesis rates also being obtained (Supplementary Figure S2B). Thus, it can be concluded that whereas energy regeneration by endogenous enzymes in the $V$. natriegens extract is possible; this strategy is less productive than exogenous energy regeneration by creatine kinase. This may be due to the high activity of metabolic pathways unfavorably channeling glycolytic substrates away from ATP synthesis. Our finding is similar to that observed in other cell-free systems, such as upon blocking the conversion of pyruvate to PEP 
by phosphoenolpyruvate synthetase using oxalate increased productivity in an E. coli CFPS system (Kuem et al., 2006). Whether a similar increase in productivity can be achieved in the $V$. natriegens system, for example by blocking certain metabolic pathways, remains to be investigated.

\section{Prevalent Amino Acid Degradation in the V. natriegens CFPS system}

The supply of the translation reaction with amino acids has previously been shown to be critical in cell-free systems (Kim and Choi, 2000; Jewett and Swartz, 2004b; Calhoun and Swartz, 2006; Kim et al., 2006). A bottleneck in the amino acid supply can partially be overcome by raising the amino acids concentration up to $2 \mathrm{mM}$ (Kim and Swartz, 2001) or by designing strains lacking several amino acid degradation pathways (Calhoun and Swartz, 2006). As it can be assumed that amino acid degradation is prevalent in our reaction, we investigated the amino acid stability in the CFPS system from $V$. natriegens. Therefore, samples were taken after $3 \mathrm{~h}$ of reaction time and the amino acids content was determined via high performance liquid chromatography (HPLC) analysis and compared to initial conditions. In the reaction system empowered by creatine phosphate, the three amino acids aspartic acid, serine, and arginine were almost entirely consumed (Figure 3A). Notably, aspartic acid was entirely depleted in the reaction, whereas more than half of the amounts of serine and arginine were degraded. A similar scenario was obtained for the system fueled by PEP, wherein it was also demonstrated that aspartic acid, serine, and arginine could be regarded as most critical (Figure 3B). Moreover, the amount of alanine doubled in the reaction fueled by PEP. It may be hypothesized that several enzymatic activities are responsible for the observed turnover of amino acids. For example, it was previously demonstrated that arginine depletion was due to arginine decarboxylase activity in a CFPS system from E. coli (Calhoun and Swartz, 2006). In addition, it was claimed that the decrease of serine is associated with pyruvate formation by serine deaminase (Calhoun and Swartz, 2006). It is therefore likely that similar enzymatic activities are present in the cell-free extract from $V$. natriegens as in E. coli, indicating a similar core metabolism as has been recently demonstrated (Long et al., 2017).

\section{Transcription Template Source Impacts Translational Performance}

For transcription of eGFP we used our standard expression plasmid, which we prepared from E. coli DH5 $\alpha$. However, to test whether the plasmid source affected the transcriptional and translational performance, we purified the plasmid from $V$. natriegens and compared the performance of CFPS reactions

A

Asp Glu Asn Ser His Gly Thr Arg Ala Tyr Gln Val Met Trp ile leu Phe Lys Pro

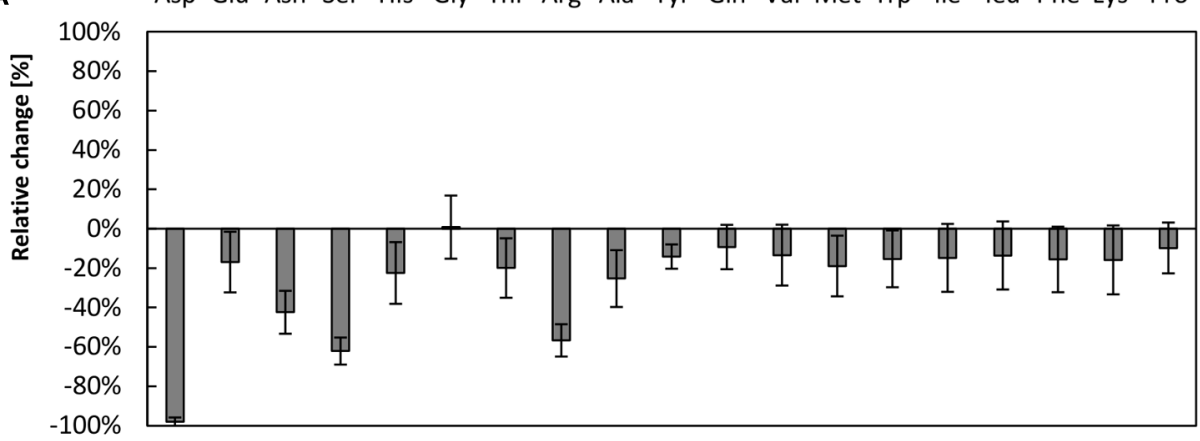

B Asp Glu Asn Ser His Gly Thr Arg Ala Tyr Gln Val Met Trp ile Phe Leu Lys Pro

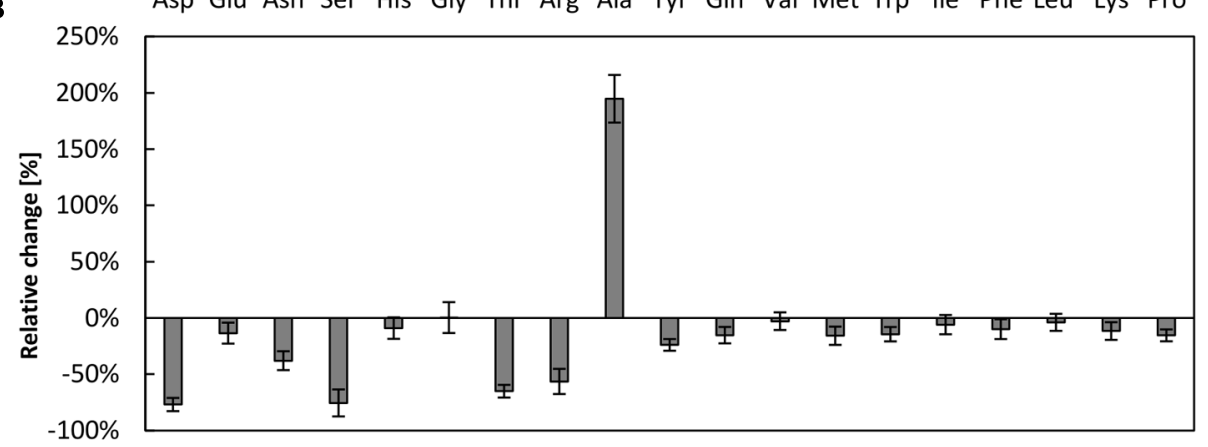

FIGURE 3 | Amino acid degradation in the $V$. natriegens CFPS system. Relative concentrations of amino acids after $3 \mathrm{~h}$ of CFPS fueled by (A) creatine phosphate or (B) PEP. Initial concentrations were set to $1 \mathrm{mM}$ of each amino acid. Note that cysteine could not be reliably measured using our analysis method. Error bars represent standard deviations of triplicate measurements. 


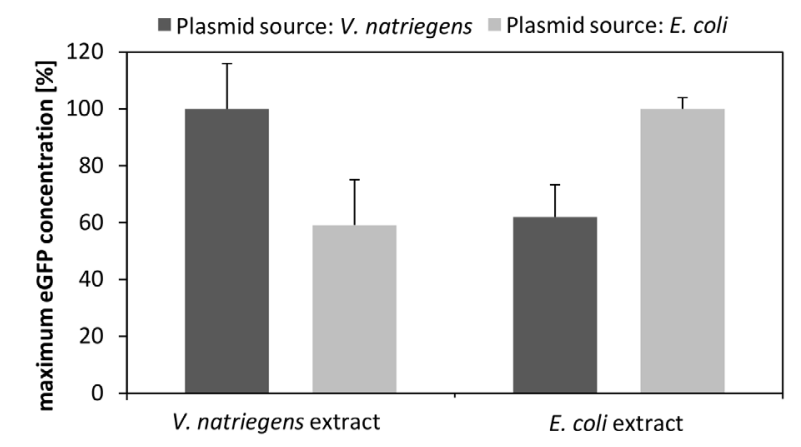

FIGURE 4 | CFPS performed with $V$. natriegens cell-free extract and identical plasmids prepared from $V$. natriegens or E. coli. The same experiment was also performed with $E$. coli extract and plasmids prepared either from $V$. natriegens or from E. coli. The maximum eGFP concentration obtained after $3 \mathrm{~h}$ of an in vitro translation reaction is shown. Plasmid concentrations were kept constant for all experiments. with that using the plasmid prepped from E. coli. As depicted in Figure 4, the source of the plasmid markedly affected the translation performance. Specifically, the $V$. natriegens CFPS system performed $40 \%$ better in the case where the plasmid was also prepared from $V$. natriegens. Notably, the control experiment using our established E. coli CFPS system with the plasmids from each source confirmed our findings. It can be hypothesized that differences in the restriction-modification system between both species impact the plasmid stability and subsequently the transcription and translation performance. This is emphasized by the fact that the transformation efficiency of $V$. natriegens is reduced if the plasmid is isolated from a different source organism (e.g., E. coli) (Weinstock et al., 2016). Therefore, it could be concluded that it is essential that the expression plasmid have the same cellular background as the CFPS systems to which it is applied.

\section{The V. natriegens Core RNAP Can Be Recruited for CFPS in Vitro}

A powerful alternative to the common transcription strategy by exogenous T7 RNAP is the recruitment of the core RNAP, which comprises part of the cell-free extract. It has been demonstrated that the core RNAP together with the housekeeping factor $\sigma^{70}$ acts as a powerful transcription unit in a CFPS system based on E. coli (Shin and Noireaux, 2010). Therefore, we chose to test whether a similar transcription unit could be activated in our $V$. natriegens system. Thus, the reaction was supplemented with a readily available plasmid in which eGFP expression was controlled by the $\sigma^{70-}$ dependent $\mathrm{Pr}$ promoter originating from $E$. coli. Considering the fundamental similarities between the two systems described above, we reasoned that this promoter sequence could also function in the $V$. natriegens system. Comparison of CFPS by the core RNAP to the system in which mRNA was generated by T7 RNAP showed identical titers of eGFP (Figure 5). Notably, reaction kinetics appeared to be faster in the system where mRNA was generated by the core RNAP. Conversely, it was previously demonstrated that the T7

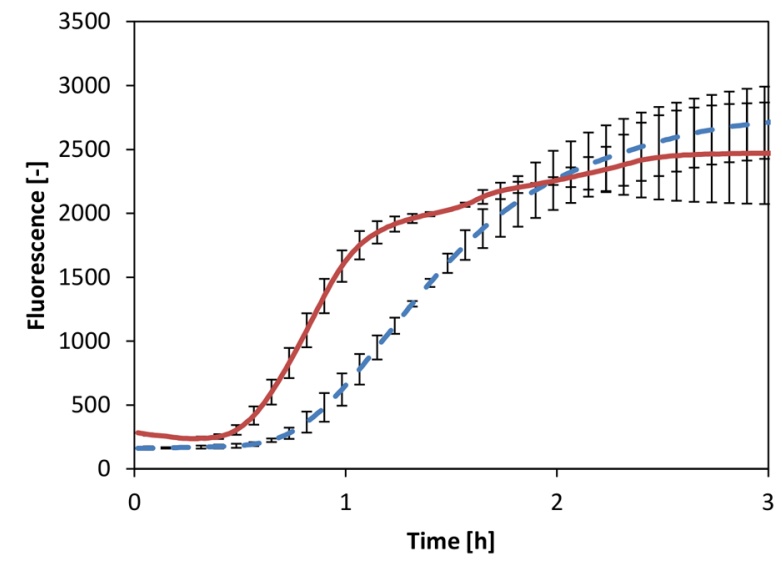

FIGURE 5 | CFPS with T7 RNAP (dotted line) and core RNAP (solid line). Error bars represent standard deviations of triplicate measurements.

RNAP is quicker than the core RNAP, at least in reported CFPS systems based on E. coli extract, resulting in an uncoupling of transcription and translation (Iskakova et al., 2006). However, we considered that the use of core RNAP might lead to extended synchronization of transcription and translation events, thus enabling more efficient translation in earlier reaction times, as was observed. Overall, this experiment clearly demonstrated that the $V$. natriegens core RNAP could be recruited to enable protein expression in vitro.

\section{rRNA Stability in the CFPS Reaction Requires Magnesium Homeostasis}

rRNA is a potential target for degradation in the CFPS reaction owing to sequestration of magnesium ions, as previously demonstrated for a CFPS system from E. coli (Failmezger et al., 2016). This motivated us to evaluate the rRNA stability in the $V$. natriegens system. Analysis of the rRNA in a CFPS reaction fueled with creatine phosphate demonstrated a decay of intact $16 \mathrm{~S}$ and $23 \mathrm{~S}$ rRNA (Figure 6A), consistent with the accompanying decrease of magnesium as shown in our previous study (Failmezger et al., 2016). In contrast, providing magnesium homeostasis by using pyruvate resulted in elevated stability of both rRNA species (Figure 6B). Based on these observations, it was evident that the CFPS system from $V$. natriegens is prone to similar rRNA degradation mechanisms as reported for E. coli (Failmezger et al., 2016). However, although the $16 \mathrm{~S}$ rRNA represented the main target for degradation in the E. coli CFPS system, the experimental results presented here suggested an equal decay of both the $16 \mathrm{~S}$ and the $23 \mathrm{~S}$ rRNA. Moreover, the electropherograms revealed no cleavage products (Supplementary Figure S3); therefore, it could be hypothesized that the rRNA degradation can be characterized by an exonuclease activity. Thus, our efforts clearly demonstrated that magnesium also constitutes a key parameter for efficient translation and preservation of ribosome integrity in the $V$. natriegens CFPS system. 

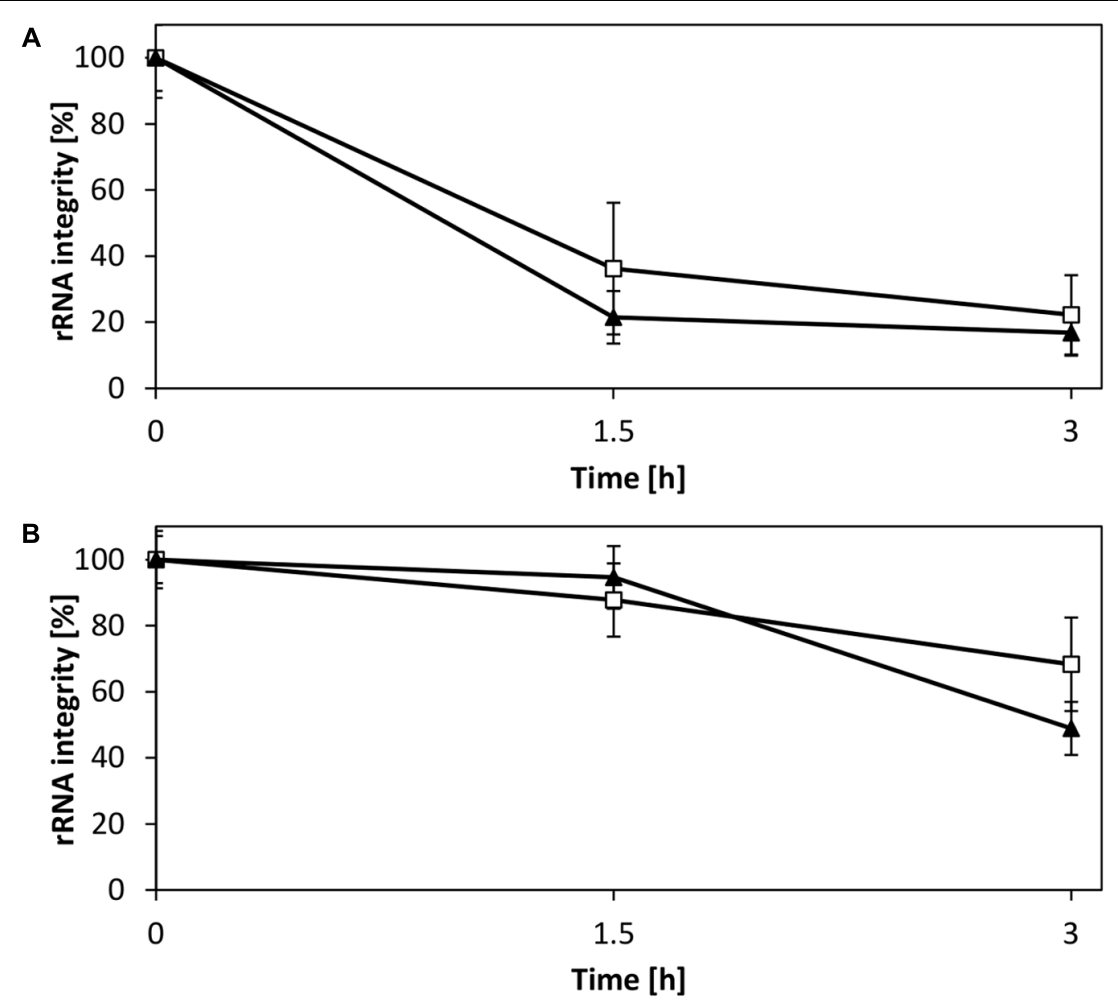

FIGURE 6 | Stability of rRNA in CFPS reactions. Shown is the integrity of the 16S rRNA ( $\square$ ) and 23S rRNA ( $\mathbf{v})$. (A) CFPS fueled with creatine phosphate, which results in the accumulation of phosphate and magnesium sequestration. (B) CFPS fueled with pyruvate allows for magnesium homeostasis and demonstrates elevated rRNA stability. Error bars represent standard deviations of triplicate measurements.

\section{Optimized Reaction and Scale Down}

Using the insights obtained regarding the $V$. natriegens CFPS system, we performed cell-free synthesis of eGFP under optimized reaction conditions. This included, e.g., elevated concentrations of amino acids, optimized magnesium levels, and plasmid prepped from $V$. natriegens. In this manner, we were able to synthesize up to $90 \mu \mathrm{g} / \mathrm{mL}$ eGFP at a reaction scale of $250 \mu \mathrm{L}$ (Figure 7A). However, it is evident that the reaction scale limits the productivity of CFPS systems (Voloshin and Swartz, 2005). Therefore, by scaling down the reaction, final product titers could be further increased up to four-fold (Figure 7B).

From this experiment, it was clear that the performance levels achieved thus far did not meet the expectations with respect to the measured high ribosome concentration in the extract. Although bulk protein synthesis rates for published E. coli CFPS systems are in the range of 6-10 $\mathrm{mg} \mathrm{L}^{-1} \min ^{-1}$ (Niess et al., 2017), a three- to fourfold higher ribosome concentration, as we detected in the $V$. natriegens cell-free extract, should allow for rates in the range of up to $40 \mathrm{mg} \mathrm{L}^{-1} \mathrm{~min}^{-1}$, resulting in theoretical protein levels of several grams per liter after standard reaction times. Consequently, owing to the observed still low volumetric synthesis rates, it might be inferred that only a partial fraction of the ribosomes could be activated under the current reaction conditions. Therefore, further strategies to improve the performance and to unleash the theoretical power of the system needed to be developed. In this regard, following the already established guiding principle of advancing CFPS by cytoplasmic mimicry (Jewett and Swartz, 2004a; Jewett et al., 2008 ) is expected to enable highly improved performance levels of CFPS by $V$. natriegens.

Notably, recent reports propose a similar scenario for in vitro translation systems from E. coli, wherein it was demonstrated that ribosomes were only partially actively participating in translation (Failmezger et al., 2017b; Kempf et al., 2017). Hence, it might be suggested that limited ribosome activity represents a general bottleneck in CFPS.

\section{CFPS From V. natriegens Enables Screening for Promoter Strength}

A valuable application of CFPS systems is the rapid screening for protein expression using different regulation entities (Sawasaki et al., 2002). As V. natriegens represents a potential host organism for the biotechnology industry (Hoffart et al., 2017), powerful expression systems need to be developed. A step toward this goal is the identification of strong promoters to allow efficient gene expression. To demonstrate the potential of our developed CFPS system to enable the identification of possible promoters of $V$. natriegens, we established a small promoter library consisting of a set of plasmids with three different promoter elements. Although little is known regarding promoters from $V$. natriegens, a promoter sequence for ribosomal protein P1, which is considered to be strong, has already been published 
A

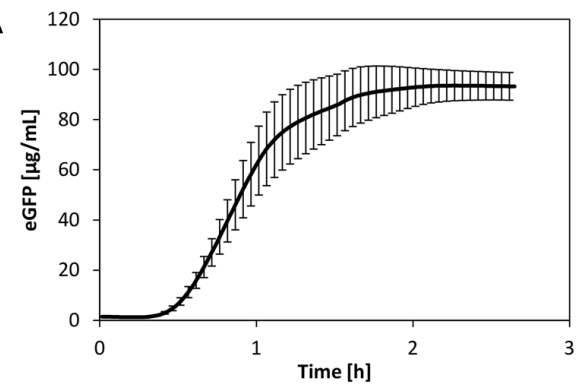

B

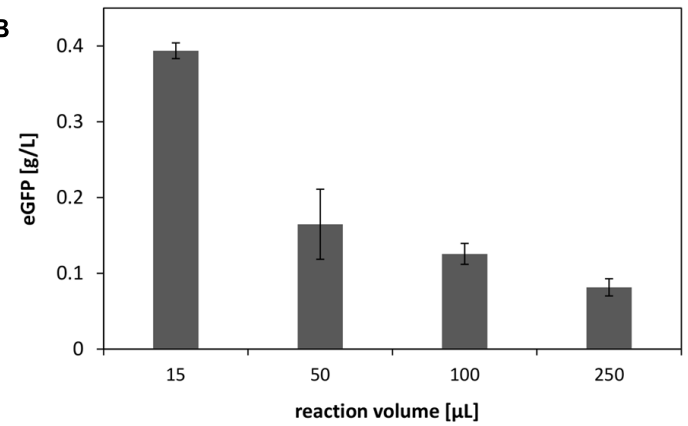

FIGURE 7 | (A) Optimized CFPS reaction of eGFP at a scale of $250 \mu \mathrm{L}$. (B) Scale down of the CFPS reaction performed in reaction tubes. Error bars represent standard deviations of triplicate measurements.

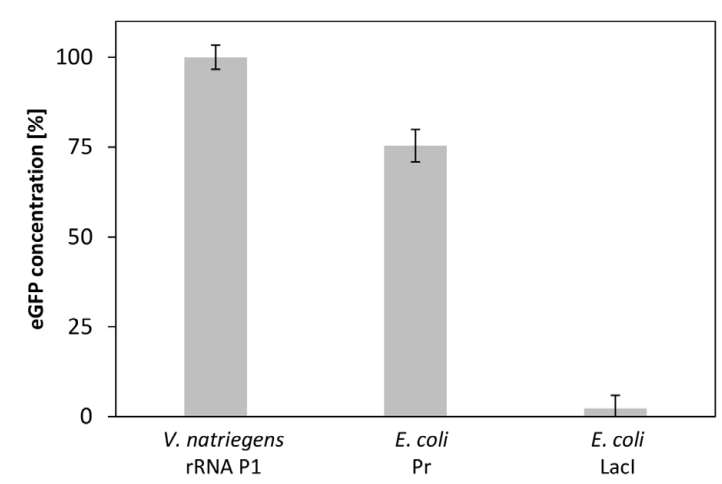

FIGURE 8 | CFPS of eGFP with $V$. natriegens extract using different promoter elements. The strong promoters rRNA P1 from $V$. natriegens and Pr from E. coli, and $\mathrm{LLaCl}$ from E. coli as a weak promoter were used. Error bars represent standard deviations of triplicate measurements.

(Aiyar et al., 2002). Lacking any information of comparable weak promoters, we relied upon the weak promoter $\mathrm{P}_{\text {lacI }}$ from E. coli. In addition, the strong $\sigma^{70}$-dependent $\mathrm{Pr}$ promoter from $E$. coli was also included in the experiment. Screening this small library showed three distinct output signals (Figure 8). Whereas the highest eGFP expression was obtained for the strong $V$. natriegens promoter, a decreased synthesis level was detected for the $\sigma^{70}$ promoter from E. coli, and almost no expression was obtained for the weak E. coli promoter. This experiment clearly demonstrated the ability to screen for promoter strength in a straightforward and rapid manner using our established CFPS system from V. natriegens.

\section{CONCLUSION}

$V$. natriegens constitutes a remarkably fast-growing bacterium that shows potential to becoming the next platform for biotechnology (Hoffart et al., 2017; Long et al., 2017). A CFPS system based on $V$. natriegens may facilitate the effective utilization of this organism for diverse applications in the fields of industrial biotechnology and synthetic biology. Herein, we reported the development of a $V$. natriegens cell-free system able to synthesize the model protein eGFP. Thorough characterization revealed the enormous potential of this system to synthesize proteins based on the measured concentration of rRNA in the cell-free extract. However, further improvements of the reaction setup and the screening of factors currently limiting the overall performance of the system, e.g., ribosome activity, is needed to exploit the full potential of this cell-free translation system. Towards this end, the application of Vibrio specific components such as tRNA, a detailed component optimization beyond E. coli specific routines (e.g., mono-and bivalent ions, buffer-specific items) and a Vibrio-specific lysate preparation routine will be initiated.

Whereas these limitations will be addressed in future work, the applicability of our $V$. natriegens cell-free protein synthesis system was successfully demonstrated by screening for potential genetic regulatory elements. Looking forward, we believe that our study represents the first step toward the establishment of a high yielding CFPS system based on extract from V. natriegens.

\section{MATERIALS AND METHODS}

\section{Culture Condition and Biomass Generation}

Vibrio natriegens was stored at $-70^{\circ} \mathrm{C}$ as a glycerol stock (30\% vol/vol). For cultivation, the stock was streaked on brain-heart infusion (BD Bactol; $\left.37 \mathrm{~g} \mathrm{~L}^{-1}\right)$ agar $\left(18 \mathrm{~g} \mathrm{~L}^{-1}\right)$ plates supplemented with $15 \mathrm{~g} \mathrm{~L}^{-1} \mathrm{NaCl}$ (BHIN-medium), and incubated overnight. A single colony was used to inoculate the preculture I $(5 \mathrm{~mL}$ BHIN-medium in a test tube. Preculture I was cultivated at $37^{\circ} \mathrm{C}$ on a rotary shaker at $120 \mathrm{rpm}$ until the stationary phase was reached. Preculture II $(100 \mathrm{~mL}$ BHIN in a $500-\mathrm{mL}$ baffled Erlenmeyer flask) was inoculated with preculture I (1/100) and cultivated for $2.5 \mathrm{~h}$ overnight at $37^{\circ} \mathrm{C}$ on a rotary shaker at $120 \mathrm{rpm}$. The main culture $(400 \mathrm{~mL}$ BHIN medium in a in a 2000-mL baffled Erlenmeyer flask) was inoculated to a cell density of 0.1 . During the cultivation, samples were taken and cell density was determined at $600 \mathrm{~nm}$ using a photometer. The differential growth rate $\mu_{\text {diff }}$ was calculated using formula (1). When the exponential phase was reached the biomass was rapidly chilled by incubation in an ice bath. Cell were harvested by centrifugation $\left(5000 \times g\right.$ for $20 \mathrm{~min}$ at $\left.4^{\circ} \mathrm{C}\right)$. Biomass was flash frozen and stored at $-70{ }^{\circ} \mathrm{C}$ until cell lysis

$$
\mu_{\mathrm{diff}}=\frac{1}{\mathrm{t} 2-\mathrm{t} 1} \times \ln \left(\frac{\mathrm{OD} 2}{\mathrm{OD} 1}\right)
$$




\section{Cell-Lysate Preparation}

Cell free lysate of $V$. natriegens was prepared according to the standard protocol that is applied in our laboratory to prepare E. coli cell free lysate. All steps aside from the run-off reaction were performed at $4{ }^{\circ} \mathrm{C}$. The frozen biomass was thawed in $1 \mathrm{~mL}$ cold S30 buffer per g biomass [ $14 \mathrm{mM}$ magnesium acetate, $60 \mathrm{mM}$ potassium acetate, $10 \mathrm{mM}$ Tris, $\mathrm{pH} 8.2,2 \mathrm{mM}$ dithiothreitol (DTT)]. The cell suspension was lysed by passage through a high-pressure homogenizer (Emulsi-Flex-C5, Avestin, Canada) at $12,000 \mathrm{kPa}$. The lysate was clarified by two centrifugation steps at $30,000 \times g$ for $30 \mathrm{~min}$ at $4^{\circ} \mathrm{C}$. Subsequently, the cell lysate was incubated at $37^{\circ} \mathrm{C}$ for $80 \mathrm{~min}$ at $120 \mathrm{rpm}$ on a shaker, for the run-off reaction. The cell-free extract was then dialyzed against a 100 -fold larger volume of S30 dialysis buffer (14 mM magnesium acetate, $60 \mathrm{mM}$ potassium acetate, $10 \mathrm{mM}$ Tris, $\mathrm{pH} 8.2,1 \mathrm{mM}$ DTT) for $4 \mathrm{~h}$ at $4^{\circ} \mathrm{C}$. After a centrifugation at $4000 \times g$ for $20 \mathrm{~min}$ at $4^{\circ} \mathrm{C}$, the clear cell lysate was aliquoted, flash frozen in liquid nitrogen, and stored at $-70^{\circ} \mathrm{C}$.

\section{CFPS}

The CFPS reaction was performed at a volume of $250 \mu \mathrm{L}$ in a 96-well plate. The standard reaction mixture consisted of the following components: $24 \%(\mathrm{v} / \mathrm{v}) \mathrm{S} 30$ cell lysate, $36 \mu \mathrm{g}$ $\mathrm{mL}^{-1}$ plasmid-DNA, $1.2 \mathrm{mM}$ of each amino acid, $18 \mathrm{mM} \mathrm{Mg}$ glutamate, $20 \mathrm{mM}$ ammonium-glutamate, $34 \mu \mathrm{g} \mathrm{mL}^{-1}$ folinic acid, 2\% (w/v) PEG (8000) $80 \mathrm{mM}$ HEPES-KOH ( $\mathrm{pH} 8.0$ ), $60 \mathrm{mM}$ creatine phosphate, $1.2 \mathrm{mM}$ ATP, $1 \mathrm{mM}$ each GTP, CTP, and UTP, $2 \mathrm{mM}$ DTT, $240 \mu \mathrm{g} / \mathrm{mL}$ creatine kinase, and 1 U/ $\mu$ L T7 RNAP (Roche Diagnostics, Mannheim, Germany). The expression of eGFP was monitored online by fluorescence (excitation filter $485 \mathrm{~nm}$, emission $520 \mathrm{~nm}$ ) in a Synergy 2 plate reader (Biotek Instruments, Winooski, VT, United States) at $37^{\circ} \mathrm{C}$. Prior to each reading cycle the plates were shaken. When PEP $(60 \mathrm{mM})$ or pyruvate $(60 \mathrm{mM})$ was used as an energy source, $0.32 \mathrm{mM} \mathrm{NAD}$ and $0.32 \mathrm{mM} \mathrm{CoA}$ were added to the reaction. The Mg-glutamate concentration was optimized for each reaction condition by performing an optimization routine consisting of a stepwise increase of $\mathrm{Mg}^{2+}$ in $2 \mathrm{mM}$ increments. The reaction was downscaled in polymerase chain reaction (PCR) tubes.

\section{rRNA Extraction and Analysis}

The extraction of the total RNA and the analysis of the rRNA from the cell lysate using capillary gel electrophoresis with laser-induced fluorescence detection was performed as described previously (Failmezger et al., 2016, 2017a).

\section{Analytics}

To quantify amino acids during the cell free reaction, samples were taken after 0,90 , and $180 \mathrm{~min}$. The samples were diluted $1 / 20$ and heated to $90^{\circ} \mathrm{C}$ for $5 \mathrm{~min}$ to stop all biochemical reactions. Precipitated proteins were removed by centrifugation ( 5 min at $5000 \times g$ and $4^{\circ} \mathrm{C}$ ) to obtain a clear supernatant.

L-amino acids were analyzed by reversed-phase HPLC using an Agilent 1200 Series apparatus (Agilent Technologies, Santa Clara, CA, United States) equipped with an Agilent
ZORBAX Eclipse Plus C18 column $(250 \times 4.6 \mathrm{~mm}, 5 \mu \mathrm{m})$ protected by a 286 Agilent ZORBAX Eclipse Plus C18 guard column $(12.5 \times 4.6 \mathrm{~mm}, 5 \mu \mathrm{m})$ and a fluorescence detector at $40^{\circ} \mathrm{C}$. The mobile phase consisted of the two eluents: Buffer A $\left(10 \mathrm{mM} \mathrm{Na} 2 \mathrm{HPO}_{4}, 10 \mathrm{mM} \mathrm{Na} \mathrm{B}_{4} \mathrm{O}_{7}, 0.5 \mathrm{mM}\right.$ $\mathrm{NaN}_{3}, \mathrm{pH}$ 8.2) and Buffer $\mathrm{B}\left[\mathrm{B} ; 0.1 \mathrm{M} \mathrm{KH}_{2} \mathrm{PO}_{4}, 0.1 \mathrm{M}\right.$ $\mathrm{K}_{2} \mathrm{HPO}_{4}, 4 \mathrm{mM}$ tetrabutylammonium sulfate, $\mathrm{pH} 7.2,30 \%$ (v/v) methanol]. Before injection, the amino acids were automatically pre-column derivatized with ortho-phthaldialdehyde and fluorenylmethoxycarbonyl chloride. The following gradient was generated at a flow rate of $1.5 \mathrm{~mL} \mathrm{~min}^{-1}: 0 \% \mathrm{~B} ; 0.84 \mathrm{~min}$, $50 \%$ B; $33.4 \mathrm{~min}, 0 \% \mathrm{~B} ; 33.5 \mathrm{~min}, 0 \%$ B $39.3 \mathrm{~min} ; 0 \% \mathrm{~B}$; $39.4 \mathrm{~min}, 0 \% \mathrm{~B} ; 40 \mathrm{~min}$. Detection of the derivatized L-amino acids occurred via a fluorescence detector, with different retention times corresponding to the single derivatized Lamino acids. The L-amino acids were quantified using an 8-point calibration curve for each amino acid as an external reference standard and by using L-ornithine as an internal standard.

\section{Plasmids}

The Plasmid pJOE4056.2 (kindly provided by J. Altenbuchner, IIG, University of Stuttgart), containing an eGFP gene between a T7-Promotor and a T7-terminator, was used for the expression of eGFP from T7-RNAP. The plasmids pJOE 4052.2 (kindly provided by J. Altenbuchner, IIG, University of Stuttgart) and pBEST-OR2-OR1-Pr-UTR1-deGFP-T500 which was a kind gift from Vincent Noireaux (Addgene plasmid \# 40019) (Shin and Noireaux, 2010) were used for the expression of eGFP from the $V$. natriegens core RNAP and $\sigma^{70}$ factor, respectively. All three plasmids contain an ampR marker for selection.

Furthermore, the Pr promoter of pBEST-OR2-OR1-Pr-UTR1deGFP-T500 was replaced by the weak promoter PLacI or the strong promoter $r R N A P 1$ using the restriction enzymes RSphI and NheI. The promoter sequences were amplified from genomic DNA of $V$. natriegens and E. coli by PCR. Restriction sites for RSphI and NheI were added using the following primer pairs $\left(5^{\prime}-3^{\prime}\right)$ : CTG GGC ATG CTA GGG GTA AAG TTG GAT AAA TAA and TAT TGC TAG CCT TCG GGA GAG GCG GCC (rRNA P1); and CTG GGC ATG CAT CGA ATG GCG CAA AAC C and TAT TGC TAG CTC ATT CAC CAC CCT GAA TTG (PLacI).

\section{DNA Template Preparation}

The plasmids were transformed into $V$. natriegens cells by electroporation and plated on BHIN-ager. Ampicillin (100 $\mu \mathrm{g} \mathrm{mL}^{-1}$ ) was used for selection during the cultivation. BHIN $(5 \mathrm{~mL})$ was inoculated with a single colony and incubated at $37^{\circ} \mathrm{C}$ and $120 \mathrm{rpm}$ until the stationary phase was reached. Then, $400 \mathrm{~mL}$ BHIN in a 2-L shake flask was inoculated $1 / 1000$ and incubated at $37^{\circ} \mathrm{C}$ and $120 \mathrm{rpm}$ overnight until the stationary phase was reached. The biomass was collected by centrifugation at $5000 \times g$ at $4^{\circ} \mathrm{C}$ for $20 \mathrm{~min}$. The DNA template for protein expression was purified using the NucleoBond ${ }^{\oplus}$ Xtra Maxi Kit (Macherey-Nagel GmbH \& Co., KG, Düren, Germany) 
according to manufacturer instruction. The DNA concentrations were measured using a NanoDrop instrument (Wilmington, DE, United States). DNA solution was diluted to $900 \mu \mathrm{g} \mathrm{mL}-1$, aliquoted, and stored at $-20^{\circ} \mathrm{C}$.

\section{AUTHOR CONTRIBUTIONS}

JF designed the study, analyzed the data, wrote and drafted the manuscript. SS performed the experiments and analyzed the data. SS, BB, and MS-H wrote sections of the manuscript and took part in designing the study. MS-H was responsible for this study.

\section{REFERENCES}

Aiyar, S. E., Gaal, T., and Gourse, R. L. (2002). rRNA promoter activity in the fast-growing bacterium Vibrio natriegens. J. Bacteriol. 184, 1349-1358. doi: 10.1128/JB.184.5.1349-1358.2002

Anastasina, M., Terenin, I., Butcher, S. J., and Kainov, D. E. (2014). Benchmarks. Biotechniques 56, 36-39.

Brödel, A. K., Sonnabend, A., and Kubick, S. (2014). Cell-free protein expression based on extracts from CHO cells. Biotechnol. Bioeng. 111, 25-36. doi: 10.1002/ bit. 25013

Calhoun, K. A., and Swartz, J. R. (2006). Total amino acid stabilization during cell-free protein synthesis reactions. J. Biotechnol. 123, 193-203. doi: 10.1016/ j.jbiotec.2005.11.011

Cordova, L. T., Long, C. P., Venkataramanan, K. P., and Antoniewicz, M. R. (2015). Complete genome sequence, metabolic model construction and phenotypic characterization of geobacillus lc300, an extremely thermophilic, fast growing, xylose-utilizing bacterium. Metab. Eng. 32, 74-81. doi: 10.1016/j.ymben.2015. 09.009

Eagon, R. G. (1962). Pseudomonas natriegens, a marine bacterium with a generation time of less than 10 minutes. J. Bacteriol. 83, 736-737.

Failmezger, J., Ludwig, J., Niess, A., and Siemann-Herzberg, M. (2017a). Quantifying ribosome dynamics in Escherichia coli using fluorescence. FEMS Microbiol. Lett. 364:fnx055. doi: 10.1093/femsle/fnx055

Failmezger, J., Rauter, M., Nitschel, R., Kraml, M., and Siemann-Herzberg, M. (2017b). Cell-free protein synthesis from non-growing, stressed Escherichia coli. Sci. Rep. 7:16524. doi: 10.1038/s41598-017-16767-7

Failmezger, J., Nitschel, R., Sánchez-Kopper, A., Kraml, M., and SiemannHerzberg, M. (2016). Site-specific cleavage of ribosomal RNA in Escherichia coli-based cell-free protein synthesis systems. PLoS One 11:e168764. doi: 10.1371/journal.pone.0168764

Gan, R., and Jewett, M. C. (2014). A combined cell-free transcription-translation system from saccharomyces cerevisiae for rapid and robust protein synthe. Biotechnol. J. 9, 641-651. doi: 10.1002/biot.201300545

Hodgman, C. E., and Jewett, M. C. (2013). Optimized extract preparation methods and reaction conditions for improved yeast cell-free protein synthesis. Biotechnol. Bioeng. 110, 2643-2654. doi: 10.1002/bit.24942

Hoffart, E., Grenz, S., Lange, J., Nitschel, R., Müller, F., Schwentner, A., et al. (2017). High substrate uptake rates empower Vibrio natriegens as production host for industrial biotechnology. Appl. Environ. Microbiol. 83, e1614-e1617. doi: 10.1128/AEM.01614-17

Iskakova, M. B., Szaflarski, W., Dreyfus, M., Remme, J., and Nierhaus, K. H. (2006). Troubleshooting coupled in vitro transcription-translation system derived from Escherichia coli cells: synthesis of high-yield fully active proteins. Nucleic Acids Res. 34, 135-144. doi: 10.1093/nar/gkl462

Jewett, M. C., Calhoun, K. A., Voloshin, A., Wuu, J. J., and Swartz, J. R. (2008). An integrated cell-free metabolic platform for protein production and synthetic biology. Mol. Syst. Biol. 4:220. doi: 10.1038/msb.2008.57

Jewett, M. C., and Swartz, J. R. (2004a). Mimicking the Escherichia coli cytoplasmic environment activates long-lived and efficient cell-free protein synthesis. Biotechnol. Bioeng. 86, 19-26.

\section{FUNDING}

The work was funded by the Bundesministerium für Bildung und Forschung (Grant Number: 031A157D, BMBF, Berlin, Germany). This publication was supported by the Open Access Publishing Fund of the University of Stuttgart.

\section{SUPPLEMENTARY MATERIAL}

The Supplementary Material for this article can be found online at: https://www.frontiersin.org/articles/10.3389/fmicb. 2018.01146/full\#supplementary-material

Jewett, M. C., and Swartz, J. R. (2004b). Substrate replenishment extends protein synthesis with an in vitro translation system designed to mimic the cytoplasm. Biotechnol. Bioeng. 87, 465-471.

Jiang, L., Zhao, J., Lian, J., and Xu, Z. (2018). Cell-free protein synthesis enabled rapid prototyping for metabolic engineering and synthetic biology. Synth. Syst. Biotechnol. 3, 90-96. doi: 10.1016/j.synbio.2018.02.003

Kelwick, R., Webb, A. J., MacDonald, J. T., and Freemont, P. S. (2016). Development of a Bacillus subtilis cell-free transcription-translation system for prototyping regulatory elements. Metab. Eng. 38, 370-381. doi: 10.1016/j. ymben.2016.09.008

Kempf, N., Remes, C., Ledesch, R., Züchner, T., Höfig, H., Ritter, I., et al. (2017). A novel method to evaluate ribosomal performance in cell-free protein synthesis systems. Sci. Rep. 7:46753. doi: 10.1038/srep46753

Kim, D.-M., and Swartz, J. R. (1999). Prolonging cell-free protein synthesis with a novel atp regeneration system. Biotechnol. Bioeng. 66, 180-188. doi: 10.1002/ (SICI)1097-0290(1999)66:3<180::AID-BIT6>3.0.CO;2-S

Kim, D.-M., and Swartz, J. R. (2001). Regeneration of adenosine triphosphate from glycolytic intermediates for cell-free protein synthesis. Biotechnol. Bioeng. 74, 309-316. doi: 10.1002/bit.1121

Kim, H.-C., and Kim, D.-M. (2009). Methods for energizing cell-free protein synthesis. J. Biosci. Bioeng. 108, 1-4. doi: 10.1016/j.jbiosc.2009.02.007

Kim, R., and Choi, C. (2000). Expression-independent consumption of substrates in cell-free expression system from Escherichia coli. J. Biotechnol. 84, 27-32. doi: 10.1016/S0168-1656(00)00326-6

Kim, T.-W., Kim, D. M., and Choi, C.-Y. (2006). Rapid production of milligram quantities of proteins in a batch cell-free protein synthesis system. J. Biotechnol. 124, 373-380. doi: 10.1016/j.jbiotec.2005. 12.030

Kuem, J.-W., Kim, W. T., Park, G. C., Choi, Y. C., and Kim, D.-M. (2006). Oxalate enhances protein synthesis in cell-free synthesis system utilizing 3phosphoglycerate as energy source. J. Biosci. Bioeng. 101, 162-165. doi: 10.1263/ jbb.101.162

Li, J., Wang, H., Kwon, C. Y., and Jewett, M. C. (2017). Establishing a high yielding streptomyces-based cell-free protein synthesis system. Biotechnol. Bioeng. 114, 1343-1353. doi: 10.1002/bit.26253

Liu, D. V., Zawada, J. F., and Swartz, J. R. (2005). Streamlining Escherichia Coli S30 extract preparation for economical cell-free protein synthesis. Biotechnol. Prog. 21, 460-465. doi: 10.1021/bp049789y

Long, C. P., Gonzalez, J. E., Cipolla, R. M., and Antoniewicz, M. R. (2017). Metabolism of the fast-growing bacterium Vibrio natriegens elucidated by 13C metabolic flux analysis. Metab. Eng. 44, 191-197. doi: 10.1016/j.ymben.2017. 10.008

Moore, S. J., Lai, E. H., Needham, H., Polizzi, K. M., and Freemont, P. S. (2017a). Streptomyces venezuelae tx-tl-a next generation cell-free synthetic biology tool. Biotechnol. J. 12:1600678. doi: 10.1002/biot.201600678

Moore, S. J., MacDonald, J. T., and Freemont, P. S. (2017b). Cell-free synthetic biology for in vitro prototype engineering. Biochem. Soc. Trans. 45, 785-791. doi: 10.1042/BST20170011

Nevin, D. E., and Pratt, J. M. (1991). A coupled in vitro transcriptiontranslation system for the exclusive synthesis of polypeptides expressed 
from the t7 promoter. FEBS Lett. 291, 259-263. doi: 10.1016/0014-5793(91) 81297-L

Niess, A., Failmezger, J., Kuschel, M., Siemann-Herzberg, M., and Takors, R. (2017). Experimentally validated model enables debottlenecking of in vitro protein synthesis and identifies a control shift under in vivo conditions. ACS Synth. Biol. 6, 1913-1921. doi: 10.1021/acssynbio.7b00117

Pardee, K., Green, A. A., Ferrante, T., Cameron, D. E., DaleyKeyser, A., Yin, P., et al. (2014). Paper-based synthetic gene networks. Cell 159, 940-954. doi: 10.1016/j.cell.2014.10.004

Pardee, K., Green, A. A., Takahashi, M. K., Braff, D., Lambert, G., Lee, J. W., et al. (2016). Rapid, low-cost detection of zika virus using programmable biomolecular components. Cell 165, 1255-1266. doi: 10.1016/j.cell.2016. 04.059

Pratt, J. (1984). "Coupled transcription-translation in prokaryotic cell-free systems," in Transcription and Translation: A Practical Approach, eds B. D. Hames and S. J. Higgins (New York, NY: IRL Press), 179-209.

Sawasaki, T., Ogasawara, T., Morishita, R., and Endo, Y. (2002). A cell-free protein synthesis system for high-throughput proteomics. Proc. Natl. Acad. Sci. U.S.A. 99, 14652-14657. doi: 10.1073/pnas.232580399

Shin, J., and Noireaux, V. (2010). Efficient cell-free expression with the endogenous E. coli RNA polymerase and sigma factor 70. J. Biol. Eng. 4:8. doi: 10.1186/17541611-4-8
Takahashi, M. K., Hayes, C. A., Chappell, J., Sun, Z. Z., Murray, R. M., Noireaux, V., et al. (2015). Characterizing and prototyping genetic networks with cell-free transcription-translation reactions. Methods 86, 60-72. doi: 10.1016/j.ymeth. 2015.05.020

Voloshin, A. M., and Swartz, J. R. (2005). Efficient and scalable method for scaling up cell free protein synthesis in batch mode. Biotechnol. Bioeng. 91, 516-521. doi: 10.1002/bit.20528

Weinstock, M. T., Hesek, E. D., Wilson, C. M., and Gibson, D. G. (2016). Vibrio natriegens as a fast-growing host for molecular biology. Nat. Methods 13, 849-851. doi: 10.1038/nmeth.3970

Conflict of Interest Statement: The authors declare that the research was conducted in the absence of any commercial or financial relationships that could be construed as a potential conflict of interest.

Copyright (C) 2018 Failmezger, Scholz, Blombach and Siemann-Herzberg. This is an open-access article distributed under the terms of the Creative Commons Attribution License (CC BY). The use, distribution or reproduction in other forums is permitted, provided the original author(s) and the copyright owner are credited and that the original publication in this journal is cited, in accordance with accepted academic practice. No use, distribution or reproduction is permitted which does not comply with these terms. 